\title{
Valproic Acid Induced Pleural Effusion: A rare Clinical and Pathophysiological Entity
}

Efthymia Papadopoulou ${ }^{1}$, Stavros Tryfon ${ }^{1, *}$, Maria Saroglou ${ }^{2}$, Dimitrios Vlachopoulos ${ }^{2}$, Athina Georgopoulou ${ }^{1}$ and Eva Serasli ${ }^{1}$

${ }^{1}$ Pulmonary Department of NHS, General Hospital "George Papanikolaou", Thessaloniki, Greece

${ }^{2}$ Private Medicine, Thessaloniki, Greece

\section{Abstract}

Introduction: Among the reported cases of valproic acid - induced pleural effusion, recurrence of pleural fluid accumulation has scarcely been presented in the literature.

Case presentation: This case study reports a 51-year-old man under treatment with valproic acid, who Published: August 22, 2020 presented with recurrent pleural effusion characterized by transition from eosinophilic to lymphocytic predominance. The recurrence of the effusion upon re-administration of the drug, along with its resolution upon discontinuation of treatment with valproic acid, is strongly indicative of a causative association.

Conclusion: This is the first reported case of recurrent valproic acid - induced pleural effusion presenting Valproic acid, Common adverse with transition from eosinophilic to lymphocytic predominance. We aim to highlight the importance of reactions, Eosinophilic pleural taking into consideration the causative role of valproic acid in inducing pleural effusion, even when the effusion, Lymphocytic pleural pleural fluid demonstrates alteration in the predominant cell type.

\section{Introduction}

This case study describes a 51-year-old man under treatment with valproic acid, who presented with recurrent pleural effusion characterized by transition from eosinophilic to lymphocytic predominance. Among the reported cases of valproic acid - induced pleural effusions, recurrence of pleural fluid accumulation has scarcely been presented in the literature. This is the first case to demonstrate alteration in the pleural fluid's predominant cell type in a patient receiving valproic acid.

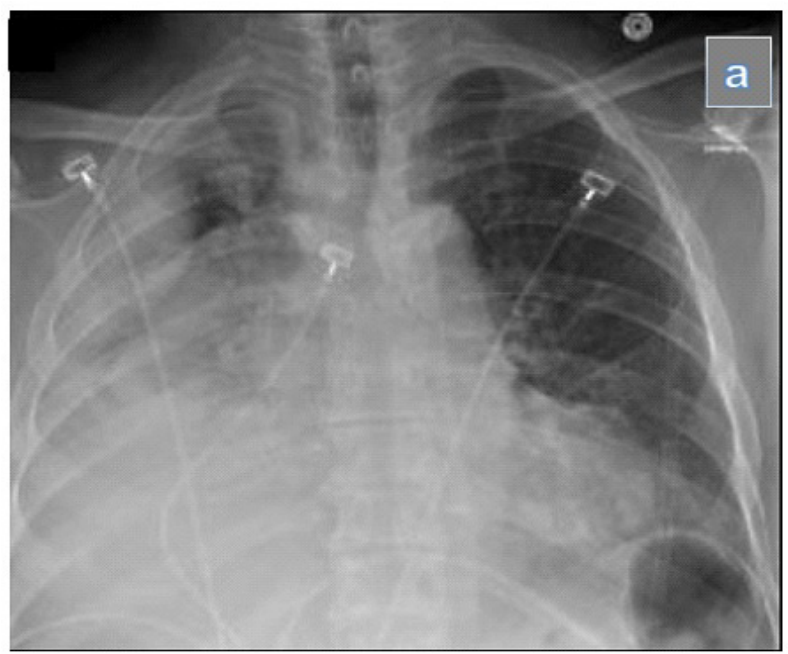

Figure 1: Initial chest X-ray showed extensive right-sided pleural effusion, which, after a diagnostic drainage was performed, presented characteristics of an eosinophilic exudate. The lung parenchyma of the right lung was revealed normal.

\section{Case Presentation}

A 51-year-old man, with a history of post-traumatic epilepsy, visited the Emergency Department complaining of progressively worsening dyspnea during the last week. He was a current smoker with a 30 packyear smoking history, and he was under antiepileptic treatment with valproic acid (500mg bid), after he had suffered craniofacial injuries in a car accident 3 years ago.
Auscultation revealed absent breath sounds in the right hemithorax and this area also presented dullness on percussion. The chest X-ray showed extensive right-sided pleural effusion (Figure 1), which, after a diagnostic drainage was performed, presented characteristics of an eosinophilic exudate (Table 1).

Cytologic examination of the pleural fluid revealed no malignant cells and acid-fast stain for mycobacterium proved negative, as did Löwenstein and Mycobacterial Growth Indicator Tube (MGIT) culture tests and Gene-Xpert. Serum biochemical as well as

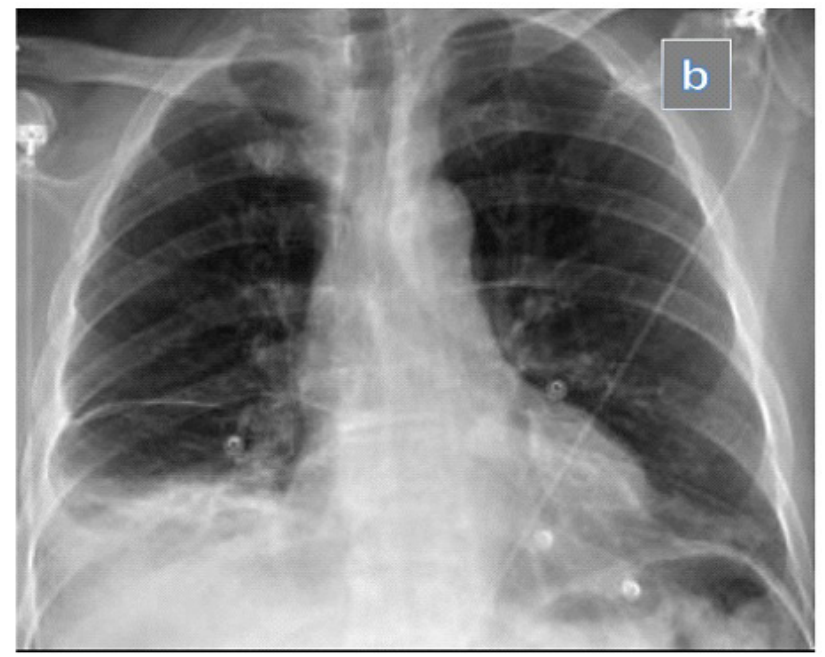

"Corresponding Author: Dr Stavros M. Tryfon, General Hospital "George E-mail: stavrostryfon@yahoo.gr

Citation: Papadopoulou E, Tryfon S, Saroglou M, Vlachopoulos D, Georgopoulou A, et al. (2020) Valproic Acid Induced Pleural Effusion: A rare Clinical and Pathophysiological Entity. Int J Clin Res Trials 5: 150. doi: https://doi. org/10.15344/2456-8007/2020/150

Copyright: (C) 2020 Papadopoulou et al. This is an open-access article distributed under the terms of the Creative Commons Attribution License, which permits unrestricted use, distribution, and reproduction in any medium, provided the original author and source are credited. 
Citation: Papadopoulou E, Tryfon S, Saroglou M, Vlachopoulos D, Georgopoulou A, et al. (2020) Valproic Acid Induced Pleural Effusion: A rare Clinical and Pathophysiological Entity. Int J Clin Res Trials 5: 150. doi: https://doi.org/10.15344/2456-8007/2020/150

Page 2 of 3

immunological tests showed no deviation from the normal values, whereas plasma concentration of valproate amounted to $72 \mathrm{mg} / \mathrm{L}$, within the therapeutic window which ranges from 50 to $100 \mathrm{mg} / \mathrm{L}$ (346-693 $\mu \mathrm{mol} / \mathrm{L})$.

After complete therapeutic drainage of the pleural fluid, the patient was discharged from the hospital with a normal chest X-ray and in good physical condition. He was advised to substitute valproic acid with levetiracetam (500mg bid). At five months of follow-up, chest $\mathrm{X}$-ray remained normal.

Seven months later the patient was referred from his neurologist because of dyspnea. By his own decision, the patient had altered his medication from levetiracetam to valproic acid two months earlier. Chest X-ray revealed accumulation of pleural fluid once again in the right hemithorax (Figure 2). Pleural fluid characteristics were evaluated anew and revealed a predominantly lymphocytic exudate (Table 1). Serum biological as well as immunological tests presented normal values once again. At the second day of his hospitalization, the patient underwent therapeutic drainage of the fluid and was discharged with a normal chest X-ray. After two months of follow-up he remains in good physical condition with normal laboratory and imaging findings.

\section{Discussion}

Recurrent pleural effusion with transition from eosinophilic to lymphocytic predominance in a patient under treatment with valproic acid after a period of discontinuation of the drug's administration has not been described in the literature until now. Although the pathophysiologic mechanism remains abstruse, the causative role of valproic acid in inducing the pleural effusion to this patient was discerned from the recurrence of the pleural effusion upon readministration of the drug and from the resolution of the effusion upon discontinuation of treatment with valproic acid. Indeed, the patient's dyspnea subsided, and no recurrence of pleural fluid accumulation was noted on follow-up after final cessation of treatment with valproic acid. Furthermore, the patient presented no other possible etiology for the development of the pleural effusion. Only two cases of recurrent pleural effusion have been attributed to treatment with valproic acid, in which, however, the fluid characteristics were preserved - one patient presenting with lymphocytic pleural effusion [1] and another with a transudative pleural effusion [2].

Valproic acid is a medication primarily used for the treatment of manic episodes in bipolar disorder, of epileptic seizures and of psychotic disorders such as schizophrenia [3], as well as for the

\begin{tabular}{|l|l|l|}
\hline Pleural Effusion Characteristics & Initial visit & Second visit (7 months later) \\
\hline Glucose $(\mathrm{mg} / \mathrm{dL})$ & 83 & 101 \\
\hline Protein $(\mathrm{g} / \mathrm{dL})$ & 6.40 & 5.77 \\
\hline Albumin $(\mathrm{g} / \mathrm{dL})$ & 3.30 & 3.47 \\
\hline LDH (U/L) & 242 & 337 \\
\hline WBC count $\left(\right.$ no. of cells $\left.\times 1,000 / \mathrm{mm}^{3}\right)$ & 2000 & 3100 \\
\hline Neutrophils & $30 \%$ & $37.5 \%$ \\
\hline Lymphocytes & $23 \%$ & $\mathbf{5 8 . 9 \%}$ \\
\hline Eosinophils & $\mathbf{4 7 \%}$ & $3.1 \%$ \\
\hline
\end{tabular}

Table 1: Characteristics of pleural fluid in a patient receiving valproic acid, who developed recurrent pleural effusion at the initial visit and 7 months later.
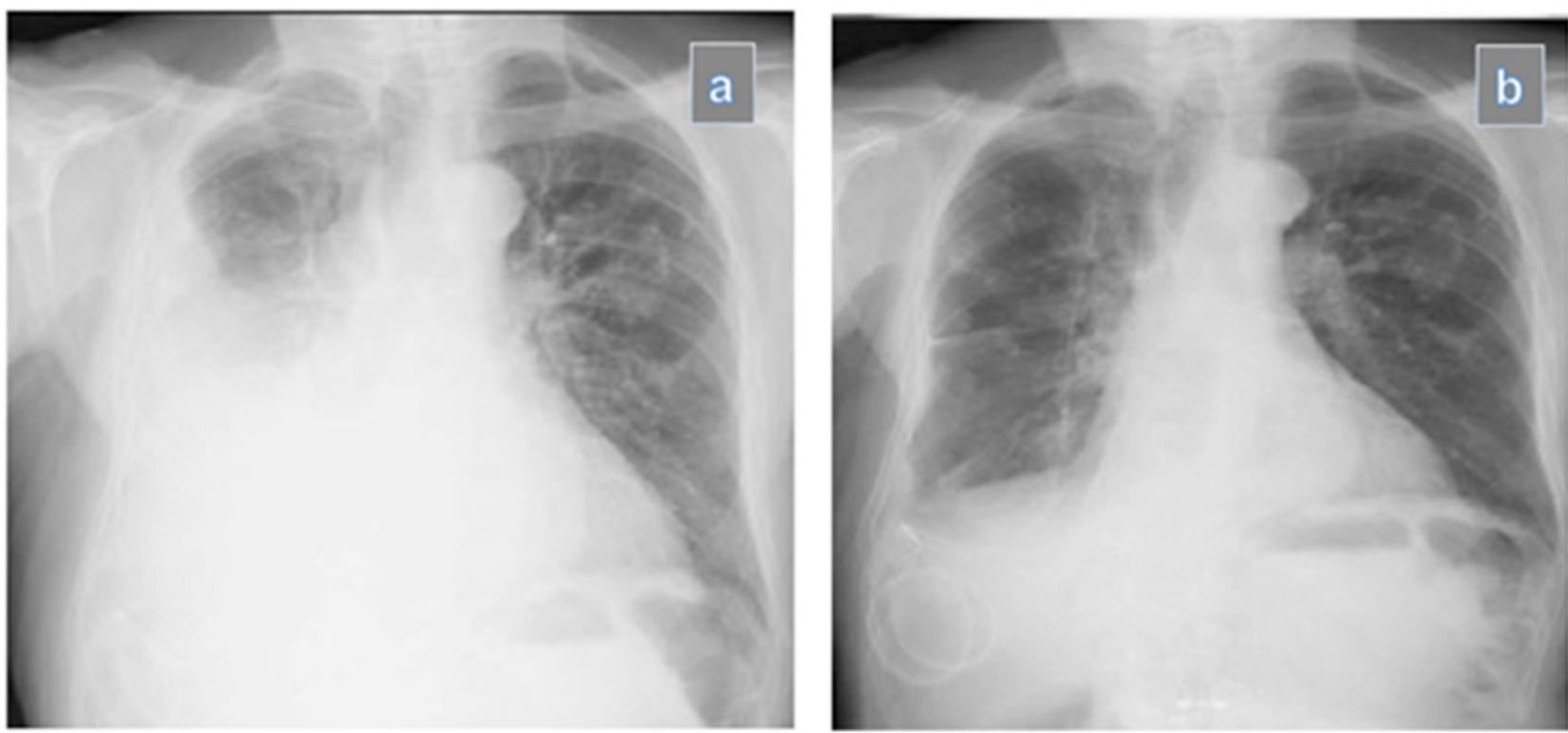

Figure 2: Chest X-ray revealed accumulation of pleural fluid once again in the right hemithorax, 7 months later. Pleural fluid characteristics were compatible with a lymphocytic exudate. After a "pleurocath" insertion, the pleural effusion resolved completely. 
Citation: Papadopoulou E, Tryfon S, Saroglou M, Vlachopoulos D, Georgopoulou A, et al. (2020) Valproic Acid Induced Pleural Effusion: A rare Clinical and Pathophysiological Entity. Int J Clin Res Trials 5: 150. doi: https://doi.org/10.15344/2456-8007/2020/150

Page 3 of 3

prevention of migraines [4]. Adverse effects commonly include nausea, vomiting, weight gain, somnolence, and tremor. Among major side effects, hepatotoxicity leading to hepatic failure, as well as potential for teratogenicity have been observed [5]. The development of pleural effusion as a result of valproic acid administration is considered a rare side effect, presenting with variable characteristics of the pleural fluid [6].

The pathophysiological processes associated with the accumulation of pleural fluid in patients receiving valproic acid have not been thoroughly clarified. In cases of pleural fluid eosinophilia, acute hypersensitivity reaction, inflammation of the pleural cavity induced by the drug, drug toxicity, and damage to mesothelial cells due to oxidants, comprise the possible pivotal mechanisms that have been implicated in the development of the pleural effusion [7]. Generally, the influx of eosinophils into the pleural cavity is orchestrated by certain chemokines secreted by mesothelial cells upon stimulation by Th2-cytokines [8]. Elevated levels of interleukin 5, interleukin 3 as well as granulocyte-macrophage colony-stimulating factor in the pleural cavity have been postulated to enhance the prolonged survival of eosinophils in the pleural fluid [9]. On the other hand, lymphocytic pleural effusions secondary to treatment with valproic acid are scarcely reported in the literature and the pathophysiologic association remains nebulous.

Adverse reactions to drugs have been implicated in a limited proportion of pleural effusions [10]. Nevertheless, it is important not to overlook the possibility of drug-induced pleural effusion after excluding other causative factors. The etiology of eosinophilic pleural effusions includes numerous pathological conditions: air or blood penetrating the pleural cavity in cases of trauma or pneumothorax, post-thoracotomy, pulmonary embolism, benign asbestos disease, tuberculosis, parasitic as well as fungal infections, connective tissue disorders, malignancy and exposure to a variety of medications [11]. Lymphocytic predominance in the pleural fluid has been associated with malignancy and tuberculosis but may as well develop in cases of rheumatoid pleurisy [12]. A meticulous diagnostic approach of this patient led to the exclusion of all the pathological conditions.

This case highlights the importance of considering valproic acid as the cause of recurrent pleural effusion even when the characteristics of the pleural fluid present a transition from eosinophilic to lymphocytic predominance. Undoubtedly, all other possible causes are to be rigorously excluded, before such an association is made. The discontinuation of treatment with valproic acid is sufficient for the resolution of the pleural effusion in such cases, as demonstrated in this patient. It is of the utmost importance to thoroughly explain to the patient the need to comply with the alteration to his treatment, in order to prevent recurrence of the pleural effusion subsequent to the patient's reluctance and adherence to his previous medication.

\section{Conclusion}

This is the first reported case of recurrent valproic acid - induced pleural effusion presenting with transition from eosinophilic to lymphocytic predominance. Recurrence of the pleural effusion upon re-administration of valproic acid, along with resolution of the effusion upon discontinuation of treatment with valproic acid, is strongly indicative of a causative association, even though the predominant cell type is not preserved. All other etiologic factors are to be rigorously excluded before such an association is made.

\section{Competing Interests}

The authors declare that they have no competing interests.

\section{References}

1. André S, Drowart A, De Bels D (2005) Lymphocytic pleural effusion associated with valproic acid. Eur J Intern Med 16: 535.

2. Tryfon S, Saroglou M, Kazanas K, Mermigkis C, Psathakis K, et al. (2009) Sodium valproate as a cause of recurrent transudative pleural effusion: a case report. J Med Case Reports 3: 51.

3. Bialer M (2012) Why are antiepileptic drugs used for nonepileptic conditions? Epilepsia 53: 26-33.

4. Aluisio AR, Zehtabchi S (2016) Valproate for Adult Migraine Prophylaxis. Am Fam Physician.

5. Zhu MM, Li HL, Shi LH, Chen XP, Luo J, et al. (2017) The pharmacogenomics of valproic acid. J Hum Genet 62: 1009-1014.

6. Vlahopoulos D, Saroglou M, Aggelis N, Kostakis E, Lykogiannis $\mathrm{S}$, et al. (2015) Differences Between the Two Types of Pleural Effusion Related to Taking Valproic Acid; Unknown Origin Pleuritis Versus Drug Induced Lupus Pleural Effusion. Chest 148: 424A.

7. Alagha K, Tummino C, Sofalvi T, Chanez P (2011) Iatrogenic eosinophilic pleural effusion. Eur Respir Rev 20: 118-120.

8. Katayama H, Yokoyama A, Kohno N, Sakai K, Hiwada K, et al. (2002) Production of eosinophilic chemokines by normal pleural mesothelial cells. Am J Respir Cell Mol Biol 26: 398-403.

9. Nakamura Y, Ozaki T, Kamei T, Kawaji K, Banno K, et al. (1993) Factors that stimulate the proliferation and survival of eosinophils in eosinophilic pleural effusion: relationship to granulocyte/macrophage colony-stimulating factor, interleukin-5, and interleukin-3. Am J Respir Cell Mol Biol 8: 605-611.

10. Huggins JT, Sahn SA (2004) Drug-induced pleural disease. Clin Chest Med 25: $141-153$.

11. Lacy M (2020) Eosinophilic pleural effusion: A case and a review. The Southwest Respiratory and Critical Care Chronicles 8: 40-46.

12. Karkhanis VS, Joshi JM (2012) Pleural effusion: diagnosis, treatment, and management. Open Access Emerg Med 4: 31-52. 